\title{
TU/e EmonONEN

\section{Iterative learning control with basis functions for media positioning in scanning inkjet printers}

\section{Citation for published version (APA):}

Bolder, J. J., Lemmen, B. P., Koekebakker, S. H., Oomen, T. A. E., Bosgra, O. H., \& Steinbuch, M. (2012). Iterative learning control with basis functions for media positioning in scanning inkjet printers. In Proceedings of the 2012 IEEE Multi-Conference on Systems and Control (MSC 2012), 3-5 October 2012, Dubrovnik, Croatia (pp. 1255-1260). Institute of Electrical and Electronics Engineers.

Document status and date:

Published: 01/01/2012

\section{Document Version:}

Accepted manuscript including changes made at the peer-review stage

\section{Please check the document version of this publication:}

- A submitted manuscript is the version of the article upon submission and before peer-review. There can be important differences between the submitted version and the official published version of record. People interested in the research are advised to contact the author for the final version of the publication, or visit the $\mathrm{DOI}$ to the publisher's website.

- The final author version and the galley proof are versions of the publication after peer review.

- The final published version features the final layout of the paper including the volume, issue and page numbers.

Link to publication

\section{General rights}

Copyright and moral rights for the publications made accessible in the public portal are retained by the authors and/or other copyright owners and it is a condition of accessing publications that users recognise and abide by the legal requirements associated with these rights.

- Users may download and print one copy of any publication from the public portal for the purpose of private study or research.

- You may not further distribute the material or use it for any profit-making activity or commercial gain

- You may freely distribute the URL identifying the publication in the public portal.

If the publication is distributed under the terms of Article 25fa of the Dutch Copyright Act, indicated by the "Taverne" license above, please follow below link for the End User Agreement:

www.tue.nl/taverne

Take down policy

If you believe that this document breaches copyright please contact us at:

openaccess@tue.nl

providing details and we will investigate your claim. 


\title{
Iterative learning control with basis functions for media positioning in scanning inkjet printers
}

\author{
Joost Bolder $^{1}$, Bas Lemmen ${ }^{1}$, Sjirk Koekebakker ${ }^{1,2}$, Tom Oomen ${ }^{1}$, Okko Bosgra ${ }^{1}$, Maarten Steinbuch ${ }^{1}$
}

\begin{abstract}
In printing systems, the positioning accuracy of the medium with respect to the print heads directly impacts print quality. In a regular document inkjet printer, the main task of the media positioning drive is to shift the medium after the printhead has finished a pass. Most media have the tendency to deform while it is being printed due to variations in temperature and moisture content. In order to improve print quality, we propose to move the medium during printing to counteract the deformation. These small scale trajectories are performed in an operating regime of which the dynamics considerably differ from the regular transportation step. Using iterative learning control with basis functions for both positioning tasks, the positioning accuracy of the drive is improved substantially; while keeping numerical cost low.
\end{abstract}

\section{INTRODUCTION}

Printing systems often need to perform similar tasks, where the dominant disturbances are of a repetitive nature. The increasing pressure on developing low-cost printing solutions, while concurrently improving print quality asks for control strategies where the repetition of tasks is exploited.

Inkjet [1], is an important technology within printing systems as it provides a versatile method to manufacture various products such as electric coils, antennas, displays, micro sensors and electronic packages, e.g. see [2], [3]. Moreover, inkjet technology is also widely used in the printing of documents. There is a lot of research in the control of the inkjet process, see [4]-[8]. Such control strategies are used to improve the print quality.

Apart from the droplet jetting accuracy, the print quality is also determined by the positioning accuracy of the substrate or medium with respect to the print heads. Typically the positioning of the medium with respect to the printhead is a mechanical control problem [9].

In this paper we focus on the control of the Media Positioning Drive (MPD) of a wide-format scanning inkjet printer. Figure 1 shows an overview of the scanning inkjet process. The medium, e.g. paper, is printed by a moving carriage, which holds the printheads. The print is made in several passes, where the medium is shifted by the MPD with a fixed step size after each pass completes. Paper tends to locally deform after a pass of the printhead, mainly due to changes in temperature and moisture content. To increase print quality, we propose to counteract the paper deformation by translating the paper while it is being printed. Moreover, the print quality can be improved further if the regular paper step is performed more accurate. Other media types, i.e.

\footnotetext{
${ }^{1}$ Eindhoven University of Technology, Dept. of Mechanical Engineering, Control Systems Technology group, P.O. Box 513, 5600 MB Eindhoven, The Netherlands, j.j.bolder@tue.nl

2 Océ Technologies, P.O. Box 101, 5900 MA Venlo, The Netherlands
}

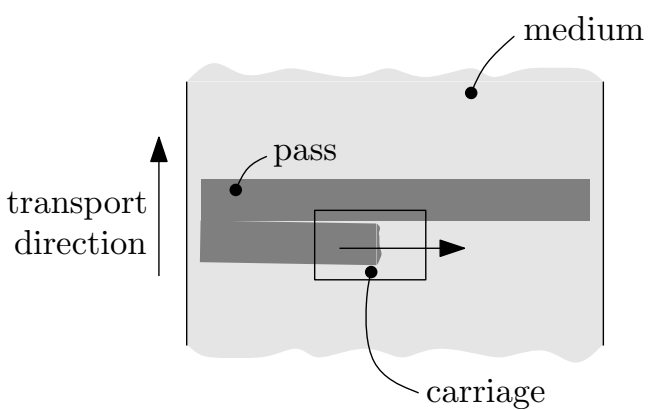

Fig. 1. Top view of the scanning inkjet printing process, showing unidirectional printing.

thin films e.g. polyamide, or fabrics also suffer from these deformations, hence the compensation strategy may also be applied for printing those materials as well.

We use the MPD for the two different tasks, the regular paper step which is a point-to-point motion task. The second task is the positioning of the paper on a micrometer scale, while it is being printed, in which transient performance is crucial. The paper deformations change every printed pass, hence in order to compensate them adequately, the microtrajectory also must vary.

Iterative Learning Control (ILC) [10]-[16] is a control strategy which can provide superior tracking performance when compared to classical linear control techniques, if the controlled system is subject to repetitive disturbances. In standard ILC it is required that the initial conditions of the process are identical every repetition [17]. Moreover, the disturbances, including the reference must be strictly identical every repetition to achieve perfect tracking. By decomposing the ILC effort intro basis functions [18], [19], which are scaled linearly with a set of parameters, it is possible to accommodate for variations in both references and initial conditions [20], which is necessary for this application.

In this paper we will derive an ILC in which both the reference and control effort are decomposed in basis functions. To handle the different operating regimes for the two tasks, two different parameterizations are kept. The controller performance is analyzed experimentally for the two positioning tasks.

This paper is organized as follows: in Section II the control problem is formulated, followed by the controller design in Section III. The experiments are elaborated on in Section IV, followed by the conclusions and outlook on future work in Section V. 


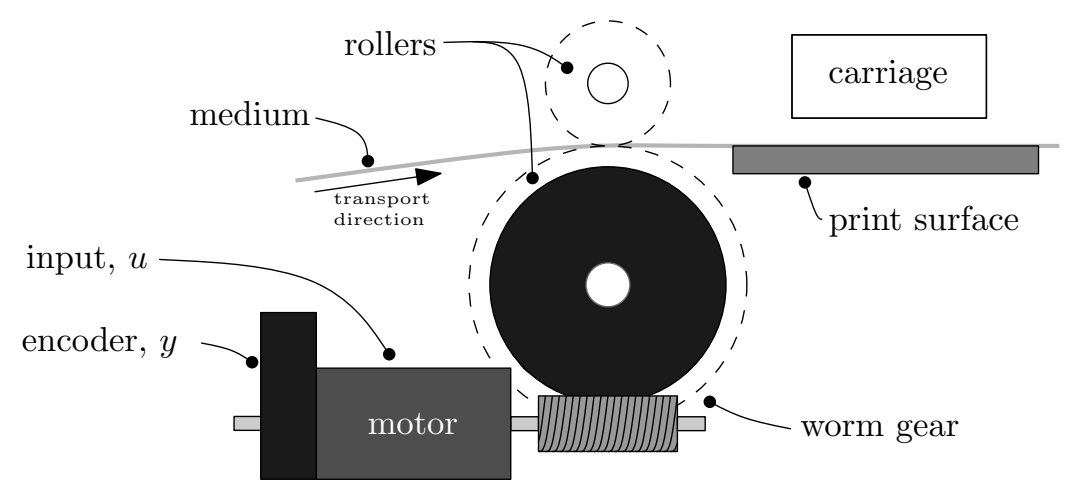

Fig. 2. Side view of the media positioning rollers. The motor is voltage driven with input $u$, the position is measured using the encoder with output $x_{1}$, the medium is positioned using two rollers, the bottom roller is driven by the motor through a worm gear. The medium is held on the print surface by a light vacuum.
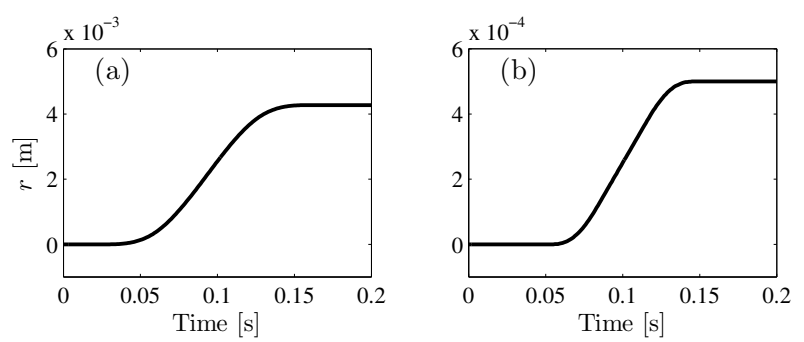

Fig. 3. Position references for the medium positioning tasks, the micro trajectory is shown in (a), the macro trajectory is shown in (b).

\section{CONTROL PROBLEM FORMULATION}

In this section, we elaborate on the control problem. The main objective is to improve print quality by using iterative learning control for two tasks. The first tasks is the regular paper shift, after a pass of the printhead completes. The second task is the tracking of a micro reference to compensate for paper deformations. For both tasks the same learning control strategy is used.

The Media Positioning Drive (MPD) is elaborated on in Fig. 2. The position of the motor is voltage controlled, the gear ratio of the worm is 60:1, such that the inertia of the motor is dominant over the inertia of the rollers. The MPD is under pretension by the paper, which exerts a force which is opposed to the indicated transport direction. This is to ensure that the drive is free from hysteresis.

The MPD has to perform two separate tasks, a regular paper step after each pass of the carriage (macro trajectory), and the tracking of a micro trajectory to compensate for paper deformation. The reference trajectories are shown in Fig. 3. For simplicity we have chosen the reference for the micro trajectory in fig Fig. $3 \mathrm{~b}$ with a similar shape as reference of the macro trajectory in Fig. 3a. This is to demonstrate the accurate tracking of this micro trajectory, without considering the actual paper deformation, and the determination of a micro reference which compensates this deformation. The references are kept constant in this paper, we do take possible variations of references into account in the controller design.

In standard ILC the initial conditions of the process and the references used must be strictly the same every repetition of the task to ensure convergence of the tracking error [17]. The reasons to use basis functions [18] in the reference construction and feedforward generation are threefold: by estimating parameters it is possible to accommodate for variations in reference trajectories, the feedforward signal is less prone to noise given that the basis functions are properly selected, and there is also a reduction of computational load and memory usage allowing trials to follow each other immediately.

The parameter update filter depends on the process model, and the feedforward basis functions. For mechanical systems, the feedforward basis functions typically depend on the reference which corresponds with compensating for inertia, friction effects, electromagnetic forces from the motor and jerk [21]. We also incorporate compensation for these effects by selecting the basis functions for the ILC as functions of the reference.

The two trajectories differ an order of magnitude in scale. We expect that due various effects, friction in particular, the tracking error will not scale linearly with the different references. Hereto, we utilize two sets of feedforward parameters, one for the macro trajectory, and one for the micro trajectory. By employing basis functions in the trajectory generation as well, a large part of the parameter update filters computation can be done à priori; the other part is computed online, which is necessary if the reference trajectory changes.

The approach in experiments is as follows: firstly the transient tracking error is improved by employing ILC with basis functions for both the macro- and micro trajectory. For the macro trajectory, the print quality is determined by the difference in tracking errors at the beginning and at the end of the task; defined as step error. The idea is, by reducing the transient tracking error, the step error is also reduced since the peak tracking error provides an upper-bound to the step error. The print quality for the micro trajectories is determined by the transient tracking error. Secondly, the performance for both trajectories is measured at the paper itself by the scanning of printed markers during the experiments. For the micro trajectory we analyze the transient performance, for the macro trajectory we analyze the step error. 


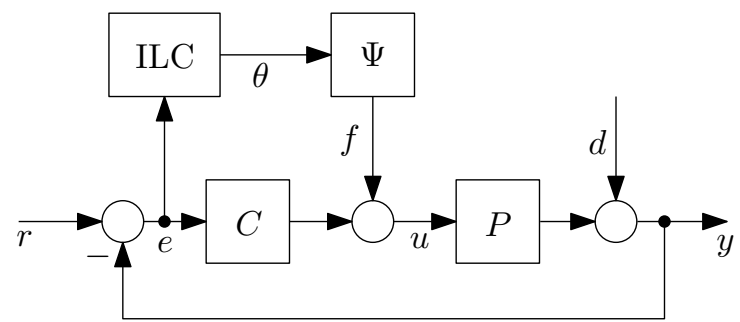

Fig. 4. Closed-loop system. The control loop consists of a standard linear feedback controller $C$ which drives plant $P$ with input $u$. The ILC updates the parameters $\theta$ at the end of each trial. The feedforward $f$ is constructed using the basis functions $\Psi$. The tracking error is $e$ which is calculated using the positioning reference $r$ and measured output $y$, which is affected by an unknown disturbance $d$.

\section{ITERATIVE LEARNING CONTROLLER DESIGN}

In this section we present the derivation of the controller, see Fig. 4 for the loop topology. Transfer functions as $P=P(z)$ are discrete-time transfer functions, we omit the argument for brevity. The error dynamics equal

$$
\begin{aligned}
e & =-P(1+P C)^{-1} f+(1+P C)^{-1}(r-d) \\
& =-S_{p} f+S \eta
\end{aligned}
$$

with $\eta=(r-d)$ external disturbances, of which the reference $r$ is known, and $d$ is unknown. The transfer function from feedforward $f$ to the tracking error $e$ is the process sensitivity $S_{p}$, the transfer from the disturbances to $e$ is the sensitivity $S$.

A trial is defined as one repetition of the reference of which the length in samples is denoted by $N$, the trial index is denoted by $k$. The tracking error $e_{k} \in \mathbb{R}^{N}$ as function of the trial index equals

$$
\begin{aligned}
e_{k} & =-S_{p} f_{k}+S \eta_{k} \\
& =-T_{0} f_{k}+T_{S} \eta_{k} .
\end{aligned}
$$

The latter matrices $T_{0} \in \mathbb{R}^{N \times N}$ and $T_{S} \in \mathbb{R}^{N \times N}$ are the lifted system representations of $S_{p}$ and $S$ respectively. Their structure is as follows:

$$
T_{x}=\left[\begin{array}{cccc}
i_{1} & & & \\
i_{2} & i_{1} & & \\
\vdots & & \ddots & \\
i_{N} & i_{N-1} & \cdots & i_{1}
\end{array}\right], \quad\left[\begin{array}{c}
i_{1} \\
i_{2} \\
\vdots \\
i_{N}
\end{array}\right]=\left[\begin{array}{c}
D \\
C B \\
\vdots \\
C A^{N-2} B
\end{array}\right]
$$

With $A, B, C$ and $D$ the respective state space matrices, empty entries are zero. The signal space of the feedforward is constructed from the basis $\Psi \in \mathbb{R}^{N \times p}$, and parameters $\theta_{k} \in \mathbb{R}^{p}$, the feedforward $f_{k} \in \mathbb{R}^{N}$ given by

$$
f_{k}=\Psi \theta_{k}
$$

Substitution of (2) in (1) yields:

$$
e_{k}\left(\theta_{k}\right)=-T_{0} \Psi \theta_{k}+T_{s} \eta_{k}
$$

The parameter update is calculated using Newton's method, which yields an convex optimization problem if the model is sufficiently close to the real system [19]. We take the weighted square of the tracking error as cost function

$$
V\left(\theta_{k}\right)=e_{k}^{T} W^{T} W e_{k},
$$

with $W \in \mathbb{R}^{N \times N}$ the weighting matrix. The gradient and Hessian are:

$$
\begin{aligned}
\nabla V\left(\theta_{k}\right) & =2 \nabla e_{k}^{T} W^{T} W e_{k}=-2 T_{0} \Psi W^{T} W e_{k}, \\
\nabla^{2} V\left(\theta_{k}\right) & =2 \nabla e_{k}^{T} W^{T} W \nabla e_{k}=2 \Psi^{T} T_{0}^{T} W^{T} W T_{0} \Psi .
\end{aligned}
$$

The parameter update by Newton optimization is:

$$
\begin{aligned}
\theta_{k+1} & =\theta_{k}-\alpha\left(\left(\nabla^{2} V\left(\theta_{k}\right)\right)^{-1} \nabla V\left(\theta_{k}\right)\right) \\
& =\theta_{k}+\alpha L e_{k}\left(\theta_{k}\right)
\end{aligned}
$$

With learning filter $L \in \mathbb{R}^{p \times N}$ in (3) and learning gain $0<\alpha<$ 2 . Note that the Hessian is completely model based, and the gradient is calculated using both the model and measurement data, the learning filter can be interpreted as the model based part.

$$
L=\left(\Psi^{T} T_{0}^{T} W^{T} W T_{0} \Psi\right)^{-1} \Psi^{T} T_{0}^{T} W^{T} W
$$

As argued in Section II, $\Psi$ is chosen as a function of the reference, hence the learning filter must be recalculated as the reference changes. By constructing the reference itself from a set of basis functions, the computational load in recalculating $L$ is reduced. Let

$$
r_{k}=R \sigma_{k}
$$

be the trial dependent reference, with basis $R \in \mathbb{R}^{N \times m}$, and parameters $\sigma_{k} \in \mathbb{R}^{m}$. Choose the feedforward basis such that it is parameterized by $\sigma_{k}$ in the following form:

$$
\Psi=\Psi_{N} \otimes \sigma_{k}
$$

With $\otimes$ the Kronecker product [22], and $\Psi_{N}$ the new basis for the feedforward. Then the gradient of the tracking error is

$$
\nabla e_{k}\left(\theta_{k}\right)=-T_{0} \Psi_{N} \otimes \sigma_{k}
$$

such that the learning filter equals:

$$
L=\left(\Psi_{N}^{T} T_{0}^{T} T_{0} \Psi_{N}\right)^{-1} \Psi_{N}^{T} T_{0}^{T} \otimes\left(\sigma_{k}^{T} \sigma_{k}\right)^{-1} \sigma_{k}^{T}
$$

with $W=I$ for brevity. Hence only the Kronecker product must be calculated online, and the largest part of this expression can be computed a priori. The signal space of the feedforward can be chosen smaller or equal to the signal space of the reference.

\section{EXPERIMENTAL RESULTS}

The experiments are performed on an Océ Colorwave 600 wide format printer. The sampling frequency is $1 \mathrm{KHz}$, the trial length $N$ is 200 samples. The design of the learning filter is discussed in the next section, followed by the elaboration on the experimental results.

\section{A. Learning filter design}

ILC requires an approximate model of the system for convergence. The model is constructed using the identification for ILC approach in [23], using the frequency domain identification algorithms in [24], [25]. Figure 5 shows the measured frequency response function of the plant, and a 4th order model. This frequency response describes the transfer from $u$ to $y$ in Fig. 2. The model includes the flexible 

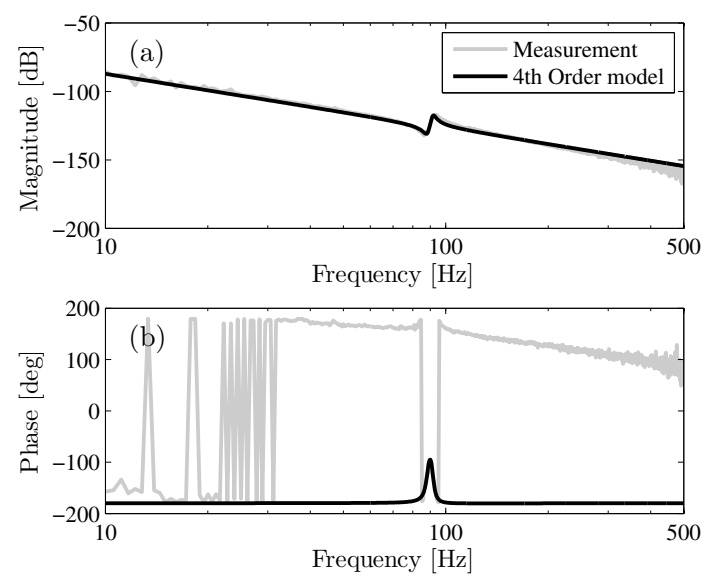

Fig. 5. Frequency response measurement of the plant with the derived 4th order model. The magnitude is shown in (a), the phase is shown in (b). There is good correspondence in the shown frequency range.
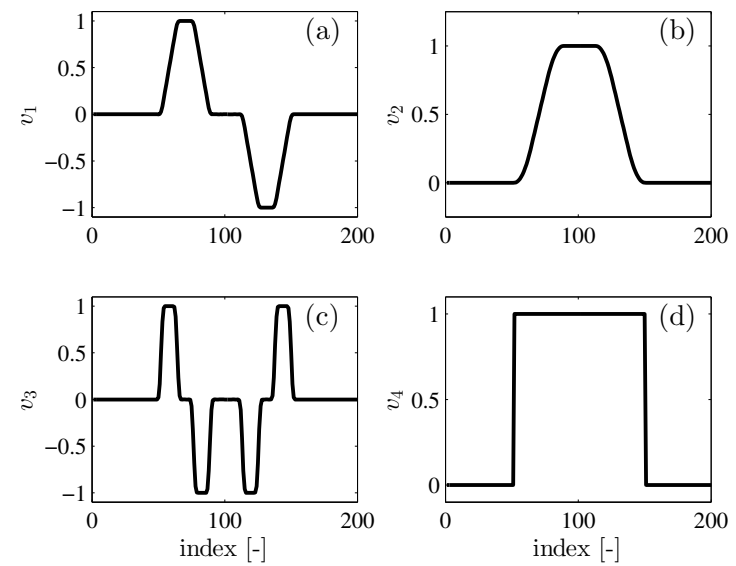

Fig. 6. The basis functions $\Psi$. Acceleration feedforward with basis function $v_{1}$ is shown in (a), velocity feedforward $v_{2}$ is shown in (b), plot (c) shows the Jerk feedforward and the basis function for Coulomb friction $v_{4}$ is shown in (d).

connection of the motor inertia with the roller inertia. The magnitude of the model coincides well, there is a difference in the phase. The model does not include delay, which is present in the physical system. The process sensitivity is calculated using the theoretical model of the controller, which in turn is used to construct $T_{0}$.

As argued in Section II, the basis functions for the feedforward are chosen as function of the reference, to compensate for electromechanical forces. The basis is chosen

$$
\Psi=\left[\begin{array}{llll}
v_{1} & v_{2} & v_{3} & v_{4}
\end{array}\right]=\left[\begin{array}{llll}
\dot{r} & \ddot{r} & \dddot{r} & \operatorname{sign}(\dot{r})
\end{array}\right],
$$

which is a common choice for motion systems [21], and is shown in Fig. 6. To improve numerical conditioning the basis functions are normalized with respect to their maximal values. Note that basis function $v_{4}$ does not fit in the form of (4), and must therefore be handled separately in the calculation of $L$, if the reference changes. So it is possible to compensate for Coulomb friction, at the expense of extra computational cost.

The weighting filter $W$ is chosen identity, such that the tracking error is penalized equally over the trial. The
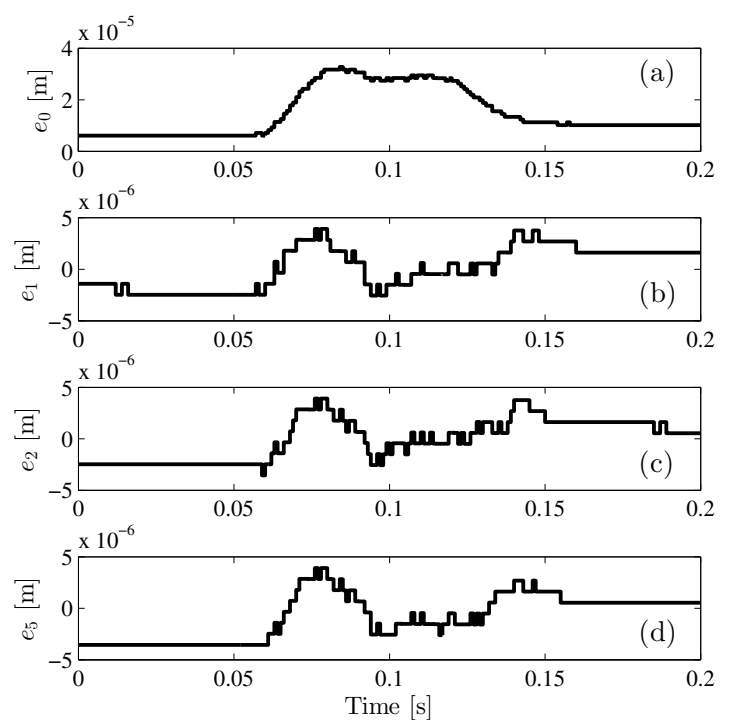

Fig. 7. Tracking errors for the micro reference for different trials. Trial 0 is shown in (a), (b) shows trial 1, and trial 2 and 5 are shown in (c) and (d) respectively. The tracking error converges after 1 trial of learning. The peak-peak error reduction is about a factor 8 .

learning gain $\alpha$ is chosen 1 . Depending on the trial varying disturbances, the learning gain can be lowered. A lower learning gain will reduce the amplification of trial varying disturbances, but it also reduces convergence speed [26]. For each task 20 trials are performed, the initial parameters $\theta_{0}$ are chosen zero. The experimental results are presented separately.

\section{B. Results of the microtrajectory task}

In Fig. 7 the tracking error for trials $0,1,2$ and 5 of the micro trajectory task are shown. In trial 0 there is no feedforward applied. The peak tracking error for the first trial is $33 \mu \mathrm{m}$. It shows that after one trial of learning the tracking error improves, the peak value is $3.9 \mu \mathrm{m}$ which is an improvement of about a factor 8 . The feedforward parameters as function of the trial number are shown in Fig. 8. It shows that it takes one trial to approximately reach their steady state value. It also shows that the parameter which corresponds to jerk feedforward is much smaller than the other parameters, which indicates that it could be omitted; as the basis functions are all normalized.

The absolute paper position is determined using markers which have been printed during the execution of the trials. By scanning the markers, the absolute paper position is determined, the result is shown in Fig. 9. Note that the alignment of the reference with the measured markers is relatively accurate for the marker position, but with limited accuracy in time. This is due to a coordinate transformation, from pixels in the scan, to the reference time. It shows the paper position for trial 0 and trial 10, a small improvement is visible, but there is a relatively large difference in final position; the paper is translated too far. A reason could be that the rollers used to transport the paper, see Fig. 2, have a rubber surface. The rollers are compressed, hence their radius is reduced. As the rollers are rotated the effective radius 

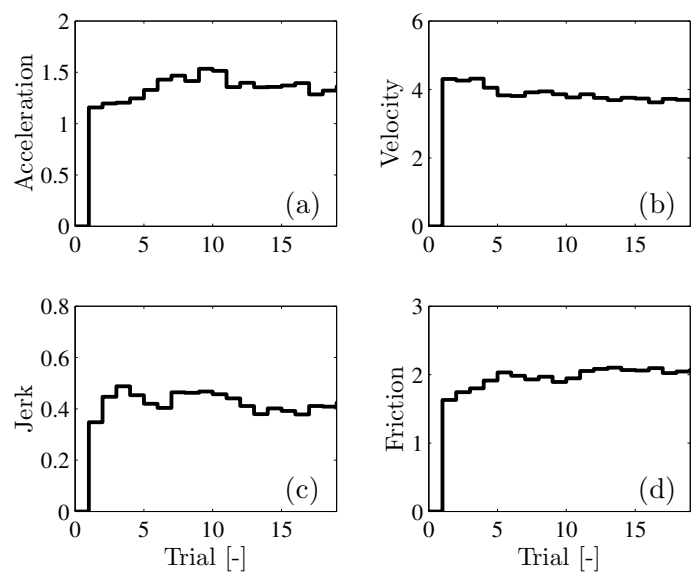

Fig. 8. The feedforward parameters for the micro trajectory as function of the trial number. It shows that the parameters converge to $90 \%$ of their final values in 1 trial.
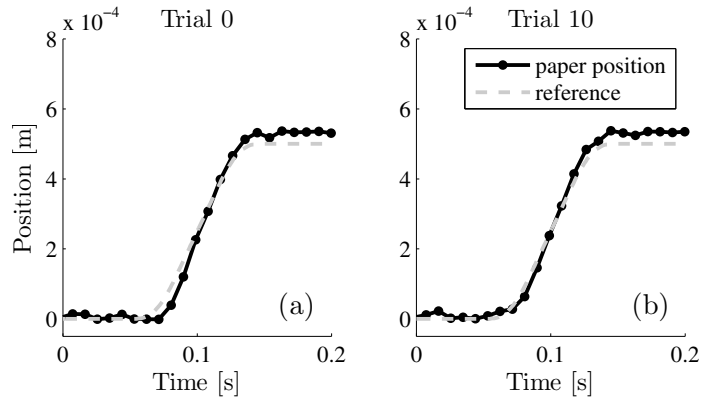

Fig. 9. Absolute paper position for the micro reference. In (a) the 0'th trial is shown, (b) shows trial 10. A small improvement in paper positioning is visible, note that the final paper position is too large for both trials.

one would calculate using the measured translation of the paper is larger than the actual radius, which is due to the incompressible nature of rubber. This implies that the gear ratio from motor to paper is depended on the compression force of the rollers.

\section{Results of the macrotrajectory task}

The results of the macro trajectory are presented in similar fashion. Figure 10 shows the tracking error for trials $0,1,2$ and 5 . The peak error of trial 0 is $102 \mu \mathrm{m}$. after 5 trials it is $5.2 \mu \mathrm{m}$, which is an improvement of a factor 20 . The tracking error converges in two trials. The parameters are shown in Fig. 11. It shows that they converge in about two trials. The coulomb friction and jerk are similar in magnitude as for the micro trajectory, but the acceleration and velocity show much larger values. The performance for the macro trajectory is determined by the step error, i.e. the difference of the tracking error at $t=0$ and $t=0.2$; which is calclated from the results shown in Fig. 10. By using printed markers, the step error measured on paper is determined. The results of comparison between the step error measured at the motor side and measured at the paper are shown in Fig. 12. It shows that the step error at the motor side is much smaller than the step error at the paper side. A small improvement is visible, but it is insignificant compared to the absolute error measured at the paper. The dominating disturbances
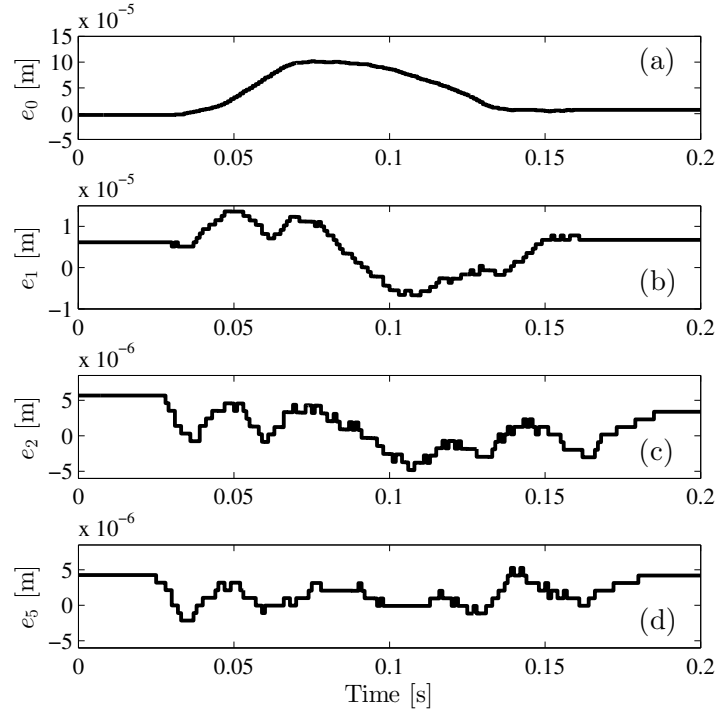

Fig. 10. Tracking errors for the macro reference for different trials. Trial 0 is shown in (a), (b) shows trial 1, and trial 2 and 5 are shown in (c) and (d) respectively. The tracking error converges after 2 trial of learning. The peak-peak error reduction is about a factor 20 .
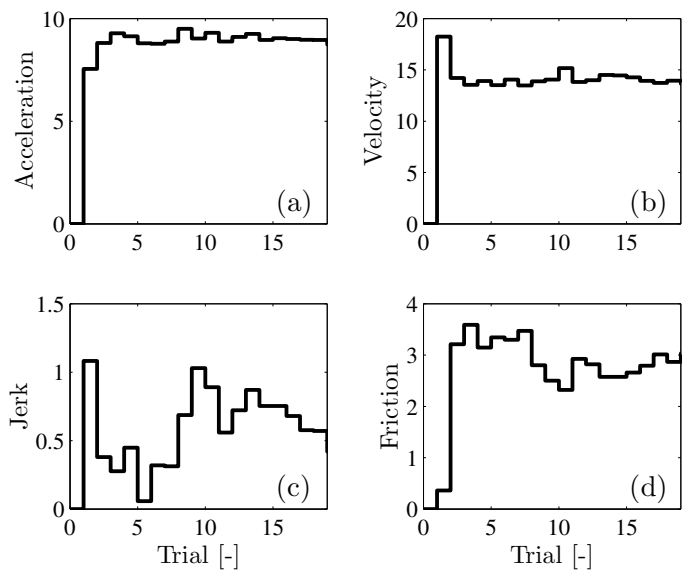

Fig. 11. The feedforward parameters for the macro trajectory as function of the trial number. It shows that the parameters converge to $90 \%$ of their final values in 2 trials.

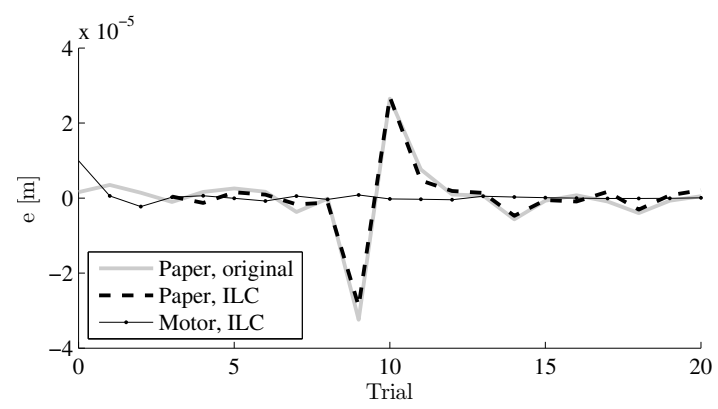

Fig. 12. Paper step error comparison. The original paper step error is shown in solid gray. The paper step error with ILC is shown in dashed black. The motor position error is shown in solid black with dots. There is a very small improvement in paper positioning error, note that the average of the error measurements on paper has been set to 0 . 
are mainly eccentricities and manufacturing tolerances of the rollers and gears. Note the large step errors at trial 9 and 10; this is caused by a deformed tooth on the worm gear, see Fig. 2. Normally no teeth are deformed, hence the peak error values can be disregarded.

\section{CONCLUSIONS AND FUTURE WORK}

The increasing pressure on more accurate printing systems demand for control strategies which exploit the repetitive nature of printing tasks. Current research in inkjet printing is mainly focussed at the control of the inkjet printheads while the positioning accuracy of the medium with respect to the printhead directly impacts the print quality, and is therefore equally important.

We focussed on improving the print quality by improving positioning accuracy of the media positioning drive. We proposed to execute small scale trajectories during printing to compensate for medium deformations. By deriving an iterative learning control strategy which enables tracking a class of references, both the tracking performance of the regular medium shifts and the tracking of the microtrajectory is improved. Analysis of the positioning accuracy of the medium revealed that the media positioning drive itself introduces disturbances which are dominant in the final positioning accuracy. Accurate tracking at the motor can be achieved as shown, however improving positioning accuracy at the medium requires further research.

An identification of the dynamic behavior from motor to the medium could provide a basis for further improvement of the medium positioning accuracy. This can be attained by utilizing an inferential control strategy, or by utilizing the markers in the feedback loop online in an iterative learning setting. In the latter case, the position of the medium is measured directly hence the dominant disturbances can be attenuated by control.

Furthermore, the tracking errors which remained after convergence of the ILC still have a lot of repetitive content; which indicates that the tracking error can be improved even further if the basis functions allow the construction of the proper feedforward. Hence, future research is the proper selection of basis functions to further improve tracking performance.

\section{REFERENCES}

[1] S. Pond, Inkjet Technology and Product Development Strategies. Torrey Pines Research, 2000.

[2] D. Mager, A. Peter, L. Tin, E. Fischer, P. Smith, J. Hennig, and J. Korvink, "An MRI Receiver Coil Produced by Inkjet Printing Directly on to a Flexible Substrate," Transactions on medical imaging, pp. 482487, 2010.

[3] J. Mujal, E. Ramon, E. Diaz, J. Carrabina, A. Calleja, R. Martinez, and L. Teres, "Inkjet Printed Antennas for NFC systems," Electronics, Circuits, and Systems, pp. 1220-1223, 2010.

[4] M. G. Wassink, O. Bosgra, and S. Koekebakker, "Enhancing inkjet printhead performance by MIMO Iterative Learning Control using implementation based basis functions," Proceedings of the American Control Conference, New York City, USA, pp. 5472-5477, 2007.

[5] A. Notenboom, D. Bruijnen, E. Homburg, R. v.d. Molengraft, L. v.d. Bedem, and M. Steinbuch, "Mechatronic design of an active printhead alignment mechanism for wide format printing systems," Mechatronics, vol. 17, pp. 109-120, 2007.
[6] D. J. Hoelzle, A. G. Alleyne, and A. J. W. Johnson, "Basis Task Approach to Iterative Learning Control with Applications to Micro-Robotic Deposition," IEEE Transactions on Control Systems Technology, vol. 19, pp. $1138-1148,2011$.

[7] D. A. Bristow and A. G. Alleyne, "A High Precision Motion Control System With Application to Microscale Robotic Deposition," IEEE Transactions On Control Systems Technology, vol. 14, pp. 1008-1020, 2006.

[8] K. L. Barton, D. J. Hoelzle, A. G. Alleyne, and A. J. W. Johnson, "Cross Coupled Iterative Learning Control of Systems with Dissimilar Dynamics: Design and Implementation for Manufacturing Applications," International Journal of Control, vol. 84, pp. 1223-1233, 2011.

[9] D. Harriman, "Control Systems Challenges in the HP Personal Ink Jet Printing Application," Proceedings of the American Control Conference, Portland, OR, USA, pp. 1138-1141, 2005.

[10] D. Bristow, M. Tharayil, and A. Alleyne, "A survey of iterative learning control: a learning-based method for high-performance tracking control," IEEE Control Systems Magazine, vol. 26, pp. 96-114, 2006.

[11] C. T. Freeman, Z. Cai, E. Rogers, and P. L. Lewin, "Iterative Learning Control for Multiple Point-to-Point Tracking Application," IEEE Transactions On Control Systems Technology, vol. 19, pp. 590-600, 2011.

[12] C. T. Freeman, "Constrained Point-to-Point Iterative Learning Control with Experimental Verification," Control Engineering Practice, vol. 20, p. $489498,2012$.

[13] R. Longman, "Iterative learning control and repetitive control for engineering practice," International Journal of Control, vol. 73, pp. 930-954, 2000.

[14] H.-S. Ahn, Y. Chen, and K. L. Moore, "Iterative Learning Control: Brief Survey and Categorization," IEEE Transactions On Systems, Man, and Cybernetics - part C: Applications and Reviews, vol. 37, pp. 1099-1121, 2007.

[15] Y. Chen, K. L. Moore, J. Yu, and T. Zhang, "Iterative Learning Control and Repetitive Control in Hard Disk Drive Industry A Tutorial," International Journal of Adaptive Control and Signal Processing, vol. 23, pp. 325-343, 2008.

[16] M. Steinbuch, J. v.d. Wijdeven, T. Oomen, K. v. Berkel, and G. Leenknegt, Model-Based Control: Bridging Rigorous Theory and Advanced Technology. Springer, 2009, ch. Recovering Data from Cracked Optical Discs using Hankel Iterative Learning Control, pp. $147-166$.

[17] K. Moore, "Iterative Learning Control: An Expository Overview," Applied and Computational Controls, Signal Processing, and Circuits, pp. $425-488,1998$.

[18] J. van de Wijdeven and O. Bosgra, "Using basis functions in iterative learning control: analysis and design theory," International Journal of Control, pp. 661-675, 2010.

[19] S. van der Meulen, R. Tousain, and O. Bosgra, "Fixed structure feedforward controller design exploiting iterative trials: application to a wafer stage and a desktop printer," Journal of Dynamic Systems, Measurement and Control: Transactions of the ASME, vol. 130, pp. $1-16,2008$.

[20] B. Lemmen, S. Koekebakker, O. Bosgra, and P. van der Bosch, "Parameterized Iterative Learning Control: Application to a wide format printer," accepted for the American Control Conference, Montreal Canada, 2012.

[21] M. Boerlage, M. Steinbuch, P. Lambrechts, and M. van de Wal, "Modelbased feedforward for motion systems," Proceedings of the IEEE Conference on Control Applications, Istanbul, Turkey, pp. 1158-1163, 2003.

[22] J. Brewer, "Kronecker Products and Matrix Calculus in System Theory," IEEE Transactions on Circuits and Systems, vol. 25, pp. 772-781, 1978.

[23] T. Oomen, J. van de Wijdeven, and O. Bosgra, "System Identification and Low-Order Optimal Control of Intersample Behavior in ILC," IEEE Transactions on Automatic Control, pp. 2734-2739, 2011.

[24] J. Schoukens, T. Dobrowiecki, and R. Pintelon, "Parametric and Nonparametric Identification of Linear Systems in the Presence of Nonlinear Distortions - A Frequency Domain Approach," IEEE Transactions On Automatic Control, vol. 43, pp. 176-190, 1998.

[25] R. Pintelon, P. Guillaume, Y. Rolain, J. Schoukens, and H. V. hamme, "Parametric Identification of Transfer Functions in the Frequency Domain - A Survey," IEEE Transactions on Automatic Control, vol. 39, 1994.

[26] D. A. Bristow and J. R. Singler, "Robustness Analysis of Slow Learning in Iterative Learning Control Systems," Proceedings of the American Control Conference, San Francisco, CA, USA, pp. 3669-3673, 2011. 